\title{
Reasonable permutation of M2e enhances the effect of universal influenza nanovaccine
}

\author{
Peiyang Ding ${ }^{1}$, Gaiping Zhang ${ }^{2}$, Yumei Chen ${ }^{1}$, Hongliang Liu ${ }^{1}$, Yunchao Liu ${ }^{3}$, Rui Jia ${ }^{4}$, \\ Yanwei Wang ${ }^{1}, \mathrm{Ge} \mathrm{Li}^{1}$, and Aiping Wang ${ }^{4}$ \\ ${ }^{1}$ Affiliation not available \\ ${ }^{2}$ Henan Academy of Agricultural Sciences \\ ${ }^{3}$ Henan Zhongze Biological Engineering Co. \\ ${ }^{4}$ Zhengzhou University
}

September 21, 2020

\begin{abstract}
Influenza A virus (IAV), a deadly zoonotic pathogen, occasionally cross-species transmission among humans, swine and avian. The ectodomain of matrix protein 2 (M2e) is highly conserved in IAV, and multi-copy M2e from different species are usually displayed on the surface of nanoparticles to improve immunogenicity and constitute universal IAV nanovaccines. In our previous study, three M2e were inserted into the C-terminal of Cap protein of porcine circovirus type 2 (PCV2) to form a universal nanovaccine that protects PCV2 and different subtypes of IAV. Howerer, M2e adopts at least two converted conformations, and the intermolecular linker of M2e enhances the conformational instability, which limits the recognition of B cell receptors and production of high-level antibodies. Here, we report that the permutation of the M2e affects immune effect of nanovaccines. Three M2e derived from humans, swine and avian IAV were inserted into the C-terminal of the Cap protein to form nanovaccines. Immunoprotective effects of different M2e arrangements were explored in mice. Results showed that the M2e closest to the surface of nanoparticle induced the most efficient protection against IAV derived from corresponding species. The results will help to develop more effective universal IAV and PCV2 bivalent nano-vaccines, as well as universal IAV vaccines for specific species.
\end{abstract}

Reasonable permutation of M2e enhances the effect of universal influenza nanovaccine

Running title: Optimize M2e universal influenza nanovaccine

Peiyang Ding ${ }^{1}$, Gaping Zhang ${ }^{1,2}$, Yumei Chen ${ }^{1}$, Hongliang Liu ${ }^{1,2}$, Yunchao Liu ${ }^{2}$, Rui $\mathrm{Jia}^{2}$, Yanwei Wang ${ }^{1,2}$, $\mathrm{Ge} \mathrm{Li}^{2}$, Aiping Wang ${ }^{1 *}$

${ }^{1}$ School of Life Sciences, Zhengzhou University, Zhengzhou 450001, China

${ }^{2}$ Henan Zhongze Biological Engineering Co., Ltd., Zhengzhou 450002, China

*To whom correspondence should be addressed:

Aiping Wang E-mail: pingaw@126.com

Tel.: +86-037167739345; Fax: +86-037163558998

Address: School of Life Sciences, Zhengzhou University, Zhengzhou 450001, China

Abstract 
Influenza A virus (IAV), a deadly zoonotic pathogen, occasionally cross-species transmission among humans, swine and avian. The ectodomain of matrix protein 2 (M2e) is highly conserved in IAV, and multi-copy M2e from different species are usually displayed on the surface of nanoparticles to improve immunogenicity and constitute universal IAV nanovaccines. In our previous study, three M2e were inserted into the C-terminal of Cap protein of porcine circovirus type 2 (PCV2) to form a universal nanovaccine that protects PCV2 and different subtypes of IAV. Howerer, M2e adopts at least two converted conformations, and the intermolecular linker of M2e enhances the conformational instability, which limits the recognition of B cell receptors and production of high-level antibodies. Here, we report that the permutation of the M2e affects immune effect of nanovaccines. Three M2e derived from humans, swine and avian IAV were inserted into the C-terminal of the Cap protein to form nanovaccines. Immunoprotective effects of different M2e arrangements were explored in mice. Results showed that the M2e closest to the surface of nanoparticle induced the most efficient protection against IAV derived from corresponding species. The results will help to develop more effective universal IAV and PCV2 bivalent nano-vaccines, as well as universal IAV vaccines for specific species.

Keywords: influenza A virus; porcine circovirus type 2; M2e; universal; nanovaccine; permutation

\section{Introduction}

Influenza A virus (IAV) causes huge economic loss to the global husbandry industry and poses a significant threat to public health. The IAV genome is composed of eight single-stranded negative-sense RNA fragments, resulting in abnormally high frequency of gene mutations and recombination, which bring great difficulties to the development of universal vaccines (Lowen, 2017). IAV occasionally cross species boundaries and pose lethal threats to other species (Long, Mistry, Haslam, \& Barclay, 2019). In particular, cross-species transmissions between humans, swine and birds are more frequent, such as 2009 pandemic H1N1 swine influenza, highly pathogenic H5N1 and H7N9 avian influenza jumped into humans, causing great panic (Gao, 2018). Therefore, it is urgent to establish IAV universal vaccines.

Hemagglutinin (HA) and neuraminidase (NA) are the main vaccine candidate antigens. However, due to the abnormally high mutation rate, current influenza vaccines can only provide effective protection against epidemic strains consistent with their HA and NA. Ectodomain of matrix protein 2 (M2e) is the most conservative and protective viral antigen and can generate hetero-subtype immunity against multiple virus strains and subtypes. However, M2e is difficult to be recognized by the immune system due to the low molecular weight, the low abundance and the steric blocking by HA and NA on the surface of IAV (Kolpe, Schepens, Fiers, \& Saelens, 2017). Various effective strategies for improving M2e antibody levels have been proposed, in particular, some nanoparticle-based nanovaccines show exciting immune effects, such as ferritin, virus-like particles (VLPs), and gold nanoparticles (Kolpe et al., 2017). To further improve the level of M2especific antibodies, nanoparticles usually display multi-copy of M2e. Tandem expression of M2e from human, swine and avian IAV are the most common pattern (Deng, Chang, et al., 2018; Deng, Mohan, et al., 2018; Ding, Jin, Chen, et al., 2019; K. H. Kim et al., 2018; M. C. Kim, Lee, et al., 2013; M. C. Kim et al., 2015; M. C. Kim, Song, et al., 2013; Petukhova et al., 2013; Qi et al., 2018; Q. Wang et al., 2020; Y. Wang et al., 2020; Yong, Yeap, Ho, Omar, \& Tan, 2015).

Porcine circovirus type 2 (PCV2), one of the smallest mammalian viruses, causes pigs severe immunosuppression and huge economic losses. The unique capsid (Cap) protein of PCV2 can self-assemble to form VLPs, which is an ideal candidate for PCV2 vaccines. What is more, the Cap VLPs can carry foreign epitopes and have the potential to become nanovaccines scaffold. Our previous research shown that Cap was capable of carrying three copies of M2e at its C-terminal without impairing VLPs formation and Cap-3M2e VLPs induced high levels of M2e-specific antibodies and PCV2-specific neutralizing antibodies in mice and pigs (Ding, Jin, Chen, et al., 2019).

However, due to the uncertainty of the crystal structure of M2e and immune systems tend to efficiently recognize stable protruding domains on the surface of nanoparticles, it is worth investigating whether the permutation of M2e of IAV from different species has an impact on the immune effect of nanovaccines.

In this study, we displayed the M2e derived from IAV of humans, swine and avian in different order (six 
orders) at the C-terminal of porcine circovirus type 2 (PCV2) Cap VLPs to explore the effect of different M2e arrangement on the immune effect of the universal vaccine. Results showed that the M2e closest to surface of Cap VLPs induced the highest M2e-specific antibodies and conferred the best protection against IAV of corresponding species. This result will help to develop more efficient universal influenza vaccines.

\section{Methods}

\subsection{Expression and purification of recombinant proteins}

Triple M2e peptides derived from human (hM2e), swine (sM2e) and avian (aM2e) IAV were combined with the C-terminus of Cap protein in different arrangements using Gly-Gly-Gly-Gly linker. (Fig. 1a-1c). These sequences were inserted into the pET28a vector by BamHI and HindIII digestion enzymes, and transformed into E. coli BL21 (DE3). Then these transformed cells were induced expression at $20^{\circ} \mathrm{C}$ for $15 \mathrm{~h}$ by isopropyl$\beta$-d-thiogalactoside (IPTG) $(0.2 \mathrm{mM})$. These recombinant proteins were purified by using Ni-NTA His.Bind Resin and identified by SDS-PAGE and Western blot. The concentrations of these purified proteins were determined with a BCA protein assay kit (Solarbio, Beijing, China). The endotoxin concentrations were measured by ToxinSensor Single Tests Kit (GenScript, Piscataway, NJ, USA). The VLPs pattern was drawn from the PDB accession number 3R0R.

\subsection{Particle characteristics of these Cap-3M2e VLPs}

These Cap-3M2e proteins were dialyzed into the assembly buffer $(10 \mathrm{mM}$ Tris- $\mathrm{HCl}, 100 \mathrm{mM} \mathrm{NaCl}(\mathrm{pH}$ 8.0)) and formed VLPs. The shape, size, size distribution and zeta potential of these Cap-3M2e VLPs were characterized by transmission electron microscopy (TEM) (JEM-1400; JEOL, Tokyo, Japan) and dynamic light scattering (DLS) (Malvern, Worcestershire, UK).

\subsection{Immunization and challenge}

Female BALB/c mice (6-8 weeks old) were randomized into groups. Six groups were subcutaneously immunized with $26.6 \mu \mathrm{g}(100 \mu \mathrm{L})$ candidate vaccines, respectively (Fig. 1a). The Cap VLPs group (20 $\mu \mathrm{g}$, Cap molar equivalent) is serves as positive control. The Carbopol $971 \mathrm{P}$ as adjuvant at final concentration $1 \%$ (Ding et al., 2017). Booster immunization was performed at the 21 and 42 days post initial immunization (dpi). Serum samples were collected at $63 \mathrm{dpi}$ and stored at $-20{ }^{\circ} \mathrm{C}$ until use. Mice in each group were divided into three groups $(n=9)$. Mice were lightly anesthetized and intranasally challenged with lethal dose humans, swine and avian IAV at $64 \mathrm{dpi}$, respectively. Survival rates and body weight loss were monitored daily for 14 days post challenge (dpc). Mice were humanely euthanized if weight loss of [?] $25 \%$.

\subsection{Antibody titer detection}

M2e- and PCV2-specific antibodies in sera were determined by indirect ELISA using $1 \mu \mathrm{g} / \mathrm{mL}$ of synthesized M2e peptides (Fig. 1c) as coating antigens. Twofold serially diluted immune serum samples were added into the ELISA plates, followed by HRP-conjugated goat anti-mouse IgG. TMB was used as substrate to develop the color reaction. The highest dilution which showing over twofold OD450 readout than that of the control sample as the antibody endpoint titer. For the detection of PCV2-specific antibodies, commercial PCV2 antibody test kit (BioChek, Reeuwijk, Holland) was utilized. The ELISA plates were coated with purified and inactivated PCV2. Immunoperoxidase monolayer assay (IPMA) was used to evaluate the PCV2-specific neutralizing antibodies in sera, according to the previous method (Ding, Jin, Chen, et al., 2019). 


\subsection{Determination of IAV titers}

Four mice from each group were sacrificed at the time when the virus titers were highest in the lungs. The MDCK cell-based IPMA was used for lung virus titration as described elsewhere(Ding, Jin, Zhou, et al., 2019). Briefly, the lung tissues were homogenized with DMEM to achieve $10 \%(\mathrm{w} / \mathrm{v})$ suspension and then centrifuged to remove tissue debris. The MDCK cells $\left(5 \times 10^{4}\right.$ cells/well) were infected with $100 \mu \mathrm{L}$ of 10 -fold serially diluted lung homogenate supernatant. After incubation for $1 \mathrm{~h}$, supernatants were removed, then fresh DMEM was added. After incubation for $24 \mathrm{~h}$, these cells were fixed, followed by IPMA to determine virus titration. IAV-specific mAbs were used as primary antibodies. The virus titers were measured by TCID $_{50}$ assay using the Reed and Muench method.

\subsection{Ethics Statement}

All BALB/c mice received humane care and all animal procedures in this study were performed in accordance with the Institutional Animal Care and Use Committee (IACUC).

\subsection{Statistical analysis}

All statistics were performed using GraphPad Prism (ver 7.0) (GraphPad Software, San Diego, CA, USA). Quantitative data are reported as means \pm SEM. $\mathrm{P}$ values were determined by one-way ANOVA analysis. Statistical significance was determined at $\mathrm{p}<0.05\left(^{*}\right)$.

\section{Results}

\subsection{Characteristics of Cap-3M2e VLPs}

Six different recombinant Cap-3M2e proteins were expressed in E. coli and purified using $\mathrm{Ni}_{2}{ }^{+}$-NTA column. SDS-PAGE and Western blot showed that these recombinant Cap-3M2e proteins were successfully expressed and purified (Fig. 1d). These recombinant Cap-3M2e proteins can be recognized well with the anti-PCV2 polyclonal antibody and 14C2 monoclonal antibody (Anti-IAV M2 protein), indicating that these recombinant proteins retained the reactogenicity of M2e and the Cap protein (Fig. 1e). Endotoxin levels in these recombinant proteins were less than $0.18 \mathrm{EU} / \mathrm{mg}$.

TEM images showed that these recombinant Cap-3M2e proteins could self-assemble into VLPs (Fig. 2a). DLS test results showed that the diameter of these Cap-3M2e VLPs were larger than that of Cap VLPs (Fig. $2 \mathrm{~b})$. The zeta potential of these Cap-3M2e VLPs were lower than that of Cap VLPs, which were caused by the strong negative charge of M2e molecules (Fig. 2c). The particle size and surface charge of these Cap-3M2e VLPs are consistent, indicating that the permutation of M2e of IAV from different species does not affect the morphology and surface charge of these VLPs (Fig. 2).

\subsection{Humoral immune effects}

All groups induced consistent PCV2-specific antibodies and neutralizing antibodies, indicated that the insertion of three copies of M2e in different orders did not affect the immunogenicity of the Cap VLPs (Fig. 3a and 3b). Total M2e-specific antibodies in Cap-3M2e VLPs groups were consistent, indicated the permutation of M2e did not affect the level of total M2e-specific antibodies (Fig. 3c). However, there were significant differences in levels of antibodies against M2e derived from human, swine and avian IAV in sera of each group. The Cap-hsaM2e VLPs and Cap-hasM2e VLPs groups induced higher anti-human IAV M2e antibodies than others (Fig. 3d). Anti-human IAV M2e antibody in the Cap-hasM2e VLPs group was 7.3 times that of the Cap-sahM2e VLPs and Cap-ashM2e VLPs groups (Fig. 3d). The Cap-shaM2e VLPs and Cap-sahM2e VLPs groups induced higher anti-swine IAV M2e antibodies than other groups (Fig. 3e). Anti-swine IAV M2e antibody in the Cap-shaM2e VLPs group was 6.4 times that of the Cap-ashM2e VLPs group (Fig. 3e). Meanwhile, anti-avian IAV M2e antibodies in the Cap-ahsM2e VLPs and Cap-ashM2e VLPs were the 
highest (Fig. 3f). The anti-avian IAV M2e antibody in Cap-ahsM2e VLPs group was 8 times that of the Cap-sahM2e VLPs group (Fig. 3f).

\subsection{Protective efficacy of Cap-3M2e VLPs against IAV}

All mice immunized with Cap VLPs died by 4 to $6 \mathrm{dpc}$ with the highest virus titers in lungs and over $25 \%$ body weight loss (Fig. 4). The protective efficacy of groups Cap-hsaM2e VLPs and Cap-hasM2e VLPs were the highest among all groups, and protected all mice from lethal infection when challenged with 10 $\times \mathrm{LD}_{50}$ A/Puerto Rico/8/1934 (H1N1) (Fig. 4a-4c). Similarly, the Cap-shaM2e VLPs and Cap-sahM2e VLPs groups showed the highest protection efficacy, and conferred complete protection against $10 \times \mathrm{LD}_{50}$ of A/swine/Zhucheng/90/2014 (H1N1) (Fig. 4d-4f). The Cap-ahsM2e VLPs and Cap-ashM2e VLPs groups demonstrated the lowest body weight loss, mortality rate and lung virus titers among all groups, when challenged with $40 \mu \mathrm{L} 10^{9} \mathrm{TCID}_{50} / \mathrm{mL}$ of A/chicken/Guangzhou/GZ/2005 (H9N2) (Fig. 4g-4i).

\section{Discussion}

High variability is the most prominent feature of IAV, which brings huge difficulties to vaccine development. Thus, the elicitation of antibody responses against sequence-conserved epitopes that could protect diverse virus strains is a high priority. The M2e is a highly conserved candidate epitope in different subtypes of IAV and offers potential to develop universal vaccines, if it can be appropriately presented and sensed by host immune system. Displaying multiple copies of M2e molecules on the surface of nanoparticles is an effective means to increase the level of anti-M2e antibodies. Although M2e is conserved among IAV, there are still some differences among strains. Particularly, M2e derived from different species varies considerably (Schepens, De Vlieger, \& Saelens, 2018). Therefore, nanoparticles usually display M2e of human, swine and avian IAV in tandem to increase the broad spectrum.

VLPs, as a kind of nanoparticles with precisely defined three-dimensional structure, provide a large number of sites for M2e that can be accurately inserted (Rodriguez-Limas, Sekar, \& Tyo, 2013). The N-terminal, C-terminal and loops are usually the prominent sites of VLPs suitable for insertion into M2e. However, loops are generally flexible structures and can only tolerate peptides of limited length (Wang et al., 2018). In this study, the N-terminal of the Cap protein is located inside the VLPs, which is difficult to be recognized by BCR and is not suitable for displaying B cell epitopes. On the contrary, the C-terminal of Cap protein protrudes from the surface of Cap VLPs, and participates in the formation of linear and conformational neutralization epitopes, indicating that the C-terminus can be efficiently recognized by immune system (Khayat et al., 2011; Lekcharoensuk et al., 2004). Therefore, it can be utilized as an insertion site for multiple copies of M2e.

Although a high affinity antibody that recognizes a pathogen's protective epitope may also bind a structurally unstable peptide, immunization with the peptide is unlikely to elicit high titer protection antibodies against the pathogen. This is because upon immunization with the peptide, any B cell that bears an immunoglobulin that recognizes one of the many conformations of the peptide can be stimulated to expand. There is no possible to selectively activate and amplify those B cells bearing immunoglobulins that bind multiple conformations of the peptide. Furthermore, the flexible peptide could guide the affinity maturation of antibodies down many alternative paths. Affinity maturation of antibodies requires stable and full display of epitopes rather than transient state (Dormitzer, Ulmer, \& Rappuoli, 2008). M2e adopt at least two transformed conformations (Cho et al., 2016; Cho et al., 2015). In the former, M2e bound to a protective $\mathrm{mAb}$ with residues Ser2-Leu3-Leu4-Thr5-Glu6 forming a N-terminal $\beta$ turn. In the latter, M2e adopts a horseshoe-like conformation that is stabilized in its core by the tryptophan residue at position 15 . In addition, due to the existence of flexible links between different M2e, the instability of multi-copy M2e 
conformation is exacerbated. Therefore, the relatively stable one in the these M2e is more easily recognized by the B cells and eventually induces higher levels of antibodies.

In order to clarify the difference between the M2e specific antibody levels of IAV from various species, we first tested the PCV2 specific antibody levels. The neutralizing antibody level of these Cap-3M2e VLPs immunization groups were consistent with the Cap VLPs group, indicating the difference of M2e antibody level in these Cap-3M2e VLPs immunization groups was related to the arrangement of M2e but not to the Cap VLPs vector. Results showed that the M2e which near the C-terminal of Cap protein induced higher levels of species-specific anti-M2e antibodies. This is because the C-terminal conformation of the Cap protein is stable and can be efficiently recognized by the immune system, thus the conformation of M2e which near the C-terminal is more stable and can be more effectively recognized by the immune system than that of other M2e. The level of species-specific anti-M2e antibodies induced by two M2e that away from the Cterminal are low and there no significant difference. This is due to the indefinite spatial conformation of M2e and the wobble of the flexible link leading to the decline of the immune system's recognition ability. The precise mechanism by which M2e-specific antibodies provide protection is controversial. But it is recognized that the protective effect is positively correlated with the level of M2e-specific antibodies. As shown in Fig. 4, groups with the highest level of species-specific M2e antibody showed the best protective effect during the challenge experimental. In general, it is necessary to selectively display M2e of IAV of species-specific in the most prominent and relatively fixed position of nanoparticles based the immune target of universal IAV vaccines, so as to induce a more efficient immune effect. For example, when pigs inject with universal IAV nanovaccines, M2e of the swine IAV (SIV) need to be displayed in a prominent and stable position on nanoparticle.

Previous studies showed that the neutralization antibody level induced by Cap VLPs at $20 \mu \mathrm{g}$ was consistent with that of the commercial PCV2 vaccine (Ingelvac CircoFLEX®), Boehringer Ingelheim)(Ding, Jin, Chen, et al., 2019; Ding, Jin, Zhou, et al., 2019). Therefore, in this study, in order to emphasize the effect of different M2e permutations on influenza virus vaccine efficacy, we used Cap VLPs instead of the commercial PCV2 vaccine and IAV VLPs as the control group. Previous studies have clearly shown that Cap-M2e VLPs nanovaccine induce high levels of PCV2-specific neutralizing antibodies and M2e-specific antibodies in mice and pigs, and significantly reduce SIV titers in pigs' respiratory tract. Cap-3M2e VLPs nanovaccine can induce higher levels of M2e-specific antibodies than Cap-M2e VLPs nanovaccine in mice, and induce high levels of PCV2-specific neutralizing antibodies consistent with commercial vaccine in mice and pigs (Ding, Jin, Chen, et al., 2019; Ding, Jin, Zhou, et al., 2019). Based on this research, the Cap-3M2e VLPs nanovaccine can be further upgraded to Cap-shaM2e VLPs nanovaccine to increase the immune effectiveness in pigs. Based on previous research, we speculate that the Cap-shaM2e VLPs nanovaccine have the potential ability to defend against the challenge of SIV and PCV2 in pigs. Therefore, recommendations for future studies include evaluation of cellular and humoral immunity in pig model and evaluation of protection against SIV and PCV2.

\section{Conclusion}

In conclusion, we studied the effect of the permutation of three M2e peptides derived from human, swine and avian IAV on the surface of PCV2 VLPs on the immune effect of IAV universal vaccine. Results demonstrate that the M2e peptide closest to the surface of the nanoparticle induced the highest immune protection against this species of IAV. This result suggests that the M2e of IAV of a specific host should be stably displayed when designing universal nanovaccines and helps to improve the immune effect of Cap-3M2e VLPs nanovaccine for pigs.

\section{Conflict of interest}

The authors report no conflicts of interest are associated with this work. 


\section{Acknowledgments}

This study was supported by the 1125 Talent Recruitment Program of Zhengzhou.

\section{Data availability statement}

Data that support the findings of this study are available from the corresponding author upon reasonable request.

\section{References}

Cho, K. J., Schepens, B., Moonens, K., Deng, L., Fiers, W., Remaut, H., \& Saelens, X. (2016). Crystal Structure of the Conserved Amino Terminus of the Extracellular Domain of Matrix Protein 2 of Influenza A Virus Gripped by an Antibody. J Virol, 90(1), 611-615. doi:10.1128/JVI.02105-15 Cho, K. J., Schepens, B., Seok, J. H., Kim, S., Roose, K., Lee, J. H., . . Kim, K. H. (2015). Structure of the extracellular domain of matrix protein 2 of influenza A virus in complex with a protective monoclonal antibody. $J$ Virol, 89(7), 3700-3711. doi:10.1128/jvi.02576-14 Deng, L., Chang, T. Z., Wang, Y., Li, S., Wang, S., Matsuyama, S., .. . Wang, B. Z. (2018). Heterosubtypic influenza protection elicited by double-layered polypeptide nanoparticles in mice. Proc Natl Acad Sci U S A, 115(33), E7758-E7767. doi:10.1073/pnas.1805713115 Deng, L., Mohan, T., Chang, T. Z., Gonzalez, G. X., Wang, Y., Kwon, Y. M., . . W Wang, B. Z. (2018). Double-layered protein nanoparticles induce broad protection against divergent influenza A viruses. Nat Commun, 9(1), 359. doi:10.1038/s41467-017-02725-4 Ding, P., Jin, Q., Chen, X., Yang, S., Guo, J., Xing, G., . . Zhang, G. (2019). Nanovaccine Confers Dual Protection Against Influenza A Virus And Porcine Circovirus Type 2.Int J Nanomedicine, 14, 7533-7548. doi:10.2147/IJN.S218057 Ding, P., Jin, Q., Zhou, W., Chai, Y., Liu, X., Wang, Y., ... Zhang, G. (2019). A Universal Influenza Nanovaccine for "Mixing Vessel" Hosts Confers Potential Ability to Block Cross-Species Transmission. Adv Healthc Mater, 8(16), e1900456. doi:10.1002/adhm.201900456 Ding, P., Zhang, T., Li, Y., Teng, M., Sun, Y., Liu, X., . . Z Zhang, G. (2017). Nanoparticle orientationally displayed antigen epitopes improve neutralizing antibody level in a model of porcine circovirus type 2. Int $J$ Nanomedicine, 12, 5239-5254. doi:10.2147/ijn.s140789 Dormitzer, P. R., Ulmer, J. B., \& Rappuoli, R. (2008). Structure-based antigen design: a strategy for next generation vaccines. Trends Biotechnol, 26(12), 659-667. doi:10.1016/j.tibtech.2008.08.002 Gao, G. F. (2018). From "A"IV to "Z"IKV: Attacks from Emerging and Re-emerging Pathogens. Cell, 172(6), 1157-1159. doi:10.1016/j.cell.2018.02.025 Khayat, R., Brunn, N., Speir, J. A., Hardham, J. M., Ankenbauer, R. G., Schneemann, A., \& Johnson, J. E. (2011). The 2.3-angstrom structure of porcine circovirus 2. J Virol, 85(15), 7856-7862. doi:10.1128/JVI.00737-11 Kim, K. H., Kwon, Y. M., Lee, Y. T., Kim, M. C., Hwang, H. S., Ko, E. J., . . Kang, S. M. (2018). Virus-Like Particles Are a Superior Platform for Presenting M2e Epitopes to Prime Humoral and Cellular Immunity against Influenza Virus. Vaccines (Basel), 6(4). doi:10.3390/vaccines6040066 Kim, M. C., Lee, J. S., Kwon, Y. M., O, E., Lee, Y. J., Choi, J. G., . . Kang, S. M. (2013). Multiple heterologous M2 extracellular domains presented on virus-like particles confer broader and stronger M2 immunity than live influenza A virus infection. Antiviral Res, 99 (3), 328-335. doi:10.1016/j.antiviral.2013.06.010 Kim, M. C., Lee, J. W., Choi, H. J., Lee, Y. N., Hwang, H. S., Lee, J., . . . Kang, S. M. (2015). Microneedle patch delivery to the skin of virus-like particles containing heterologous M2e extracellular domains of influenza virus induces broad heterosubtypic cross-protection. J Control Release, 210, 208-216. doi:10.1016/j.jconrel.2015.05.278 Kim, M. C., Song, J. M., O, E., Kwon, Y. M., Lee, Y. J., Compans, R. W., \& Kang, S. M. (2013). Virus-like particles containing multiple M2 extracellular domains confer improved cross-protection against various subtypes of influenza virus. Mol Ther, 21 (2), 485-492. doi:10.1038/mt.2012.246 Kolpe, A., Schepens, B., Fiers, W., \& Saelens, X. (2017). M2-based influenza vaccines: recent advances and clinical potential. Expert Rev Vaccines, 16 (2), 123136. doi:10.1080/14760584.2017.1240041 Lekcharoensuk, P., Morozov, I., Paul, P. S., Thangthumniyom, N., Wajjawalku, W., \& Meng, X. J. (2004). Epitope mapping of the major capsid protein of type 2 porcine circovirus (PCV2) by using chimeric PCV1 and PCV2. J Virol, 78(15), 8135-8145. doi:10.1128/JVI.78.15.81358145.2004 Long, J. S., Mistry, B., Haslam, S. M., \& Barclay, W. S. (2019). Host and viral determinants of influenza A virus species specificity. Nat Rev Microbiol, 17(2), 67-81. doi:10.1038/s41579-018-0115-z Lowen, A. C. (2017). Constraints, Drivers, and Implications of Influenza A Virus Reassortment. Annu Rev Virol, 
4(1), 105-121. doi:10.1146/annurev-virology-101416-041726 Petukhova, N. V., Gasanova, T. V., Stepanova, L. A., Rusova, O. A., Potapchuk, M. V., Korotkov, A. V., . . . Atabekov, J. G. (2013). Immunogenicity and protective efficacy of candidate universal influenza A nanovaccines produced in plants by Tobacco mosaic virus-based vectors. Curr Pharm Des, 19(31), 5587-5600. doi:10.2174/13816128113199990337 Qi, M., Zhang, X. E., Sun, X., Zhang, X., Yao, Y., Liu, S., . . . Cui, Z. (2018). Intranasal Nanovaccine Confers Homo- and Hetero-Subtypic Influenza Protection. Small, 14(13), e1703207. doi:10.1002/smll.201703207 Rodriguez-Limas, W. A., Sekar, K., \& Tyo, K. E. (2013). Virus-like particles: the future of microbial factories and cell-free systems as platforms for vaccine development.Curr Opin Biotechnol, 24 (6), 1089-1093. doi:10.1016/j.copbio.2013.02.008 Schepens, B., De Vlieger, D., \& Saelens, X. (2018). Vaccine options for influenza: thinking small.Curr Opin Immunol, 53, 22-29. doi:10.1016/j.coi.2018.03.024 Wang, D., Zhang, S., Zou, Y., Yu, W., Jiang, Y., Zhan, Y., . . . Yang, Y. (2018). Structure-Based Design of Porcine Circovirus Type 2 Chimeric VLPs (cVLPs) Displays Foreign Peptides on the Capsid Surface. Front Cell Infect Microbiol, 8, 232. doi:10.3389/fcimb.2018.00232 Wang, Q., Zhang, Y., Zou, P., Wang, M., Fu, W., She, J., . . Wu, F. (2020). Self-Assembly M2e-Based Peptide Nanovaccine Confers Broad Protection Against Influenza Viruses.Front Microbiol, 11, 1961. doi:10.3389/fmicb.2020.01961 Wang, Y., Deng, L., Gonzalez, G. X., Luthra, L., Dong, C., Ma, Y., . . W Wang, B. Z. (2020). Double-Layered M2e-NA Protein Nanoparticle Immunization Induces Broad Cross-Protection against Different Influenza Viruses in Mice. Adv Healthc Mater, 9(2), e1901176. doi:10.1002/adhm.201901176 Yong, C. Y., Yeap, S. K., Ho, K. L., Omar, A. R., \& Tan, W. S. (2015). Potential recombinant vaccine against influenza A virus based on M2e displayed on nodaviral capsid nanoparticles. Int $J$ Nanomedicine, 10, 2751-2763. doi:10.2147/IJN.S77405

\section{Fig. 1. Characterization of these Cap-3M2e proteins.}

(a) Primary pattern structure of six recombinant Cap-3M2e. (b) M2e sequence of the human, swine and avian IAV which were used in challenging experiment. (c) Schematic illustration of Cap-3M2e VLPs nanovaccine. The VLPs model was derived from the PDB database (3R0R). (d) SDS-PAGE analysis of these purified Cap-3M2e proteins. Lane M: molecular weight markers; lane 1: Cap; lane 2: Cap-hsaM2e; lane 3: CaphasM2e; lane 4: Cap-shaM2e; lane 5: Cap-sahM2e; lane 6: Cap-ahsM2e; lane 7: Cap-ashM2e. (e) Western blot analysis of these purified Cap-3M2e protein. The upper figure shows the reactivity of the recombinant protein with PCV2 positive sera. The lower figure shows the reactivity of recombinant protein with M2e specific monoclonal antibody 14C2. Lane M: molecular weight markers; lane 1: Cap; lane 2: Cap-hsaM2e; lane 3: Cap-hasM2e; lane 4: Cap-shaM2e; lane 5: Cap-sahM2e; lane 6: Cap-ahsM2e; lane 7: Cap-ashM2e.

\section{Fig. 2. Characterization of these Cap-3M2e VLPs.}

(a) Transmission electron micrograph of these Cap-3Me VLPs. Scale bars $=100 \mathrm{~nm}$. (b) Size distribution of these Cap-3M2e VLPs $(\mathrm{n}=5)$. (c) Zeta potential of these Cap-3M2e VLPs $(\mathrm{n}=5)$.

\section{Fig. 3. Humoral immune response in vaccinated mice.}

Sera were collected 21 days after the last immunization. (a) PCV2-specific IgG level, sera samples were diluted 1:100 $(\mathrm{n}=6)$. (b) PCV2-specific neutralizing antibodies level was measured by IPMA $(\mathrm{n}=6)$. (c) M2e-specific IgG level $(n=6)$. M2e of derived from humans, swine and avian IAV were mixed in equal amounts as the detection antigen $(1 \mu \mathrm{g} / \mathrm{mL})$. (d) Human IAV M2e-specific IgG level $(\mathrm{n}=6)$. (e) Swine IAV M2e-specific IgG level ( $\mathrm{n}=6)$. (f) Avian IAV M2e-specific IgG level $(\mathrm{n}=6)$. 


\section{Fig. 4. Protective efficacy of Cap-3M2e VLPs against IAV.}

Immunized mice were challenged with lethal dose of IAV at 64 dpi. Mice body weight and survival rate changes were monitored daily for 14 days. (a) Mice body weight changes $(\mathrm{n}=5)$, (b) survival rate $(\mathrm{n}=5)$ and (c) lung virus titers $(\mathrm{n}=4)$ post $10 \times \mathrm{LD}_{50}$ of A/Puerto Rico/8/1934 (H1N1) challenge. (d) Mice body weight changes $(n=5)$, (e) survival rate $(n=5)$ and (f) lung virus titers $(n=4)$ post $10 \times L_{50}$ of A/swine/Zhucheng/90/2014 (H1N1) challenge. (g) Mice body weight changes $(\mathrm{n}=5)$, (h) survival rate $(\mathrm{n}=5)$ and (i) lung virus titers $(\mathrm{n}=4)$ post $40 \mu \mathrm{L} 10^{9} \mathrm{TCID}_{50} / \mathrm{mL}$ of $\mathrm{A} /$ chicken/Guangzhou/GZ/2005 (H9N2) challenge. $\mathrm{p}<0.05(*)$.

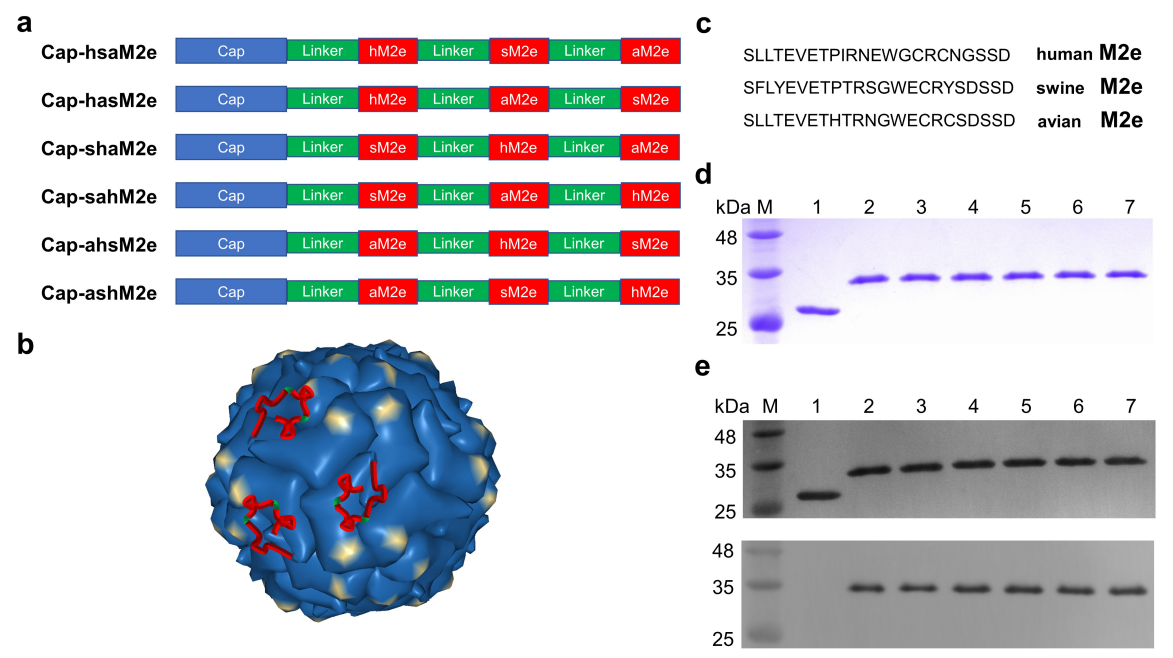

a

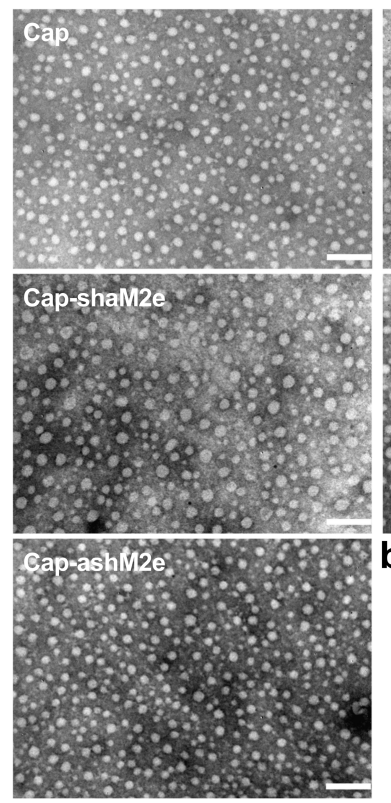

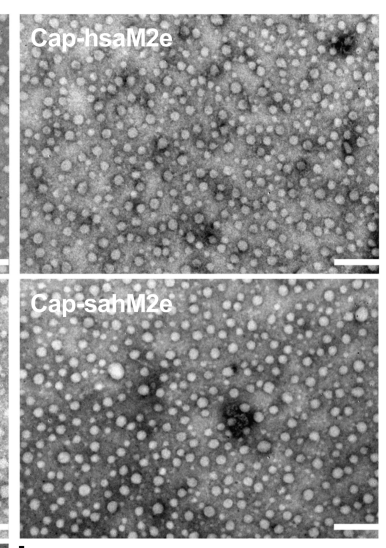

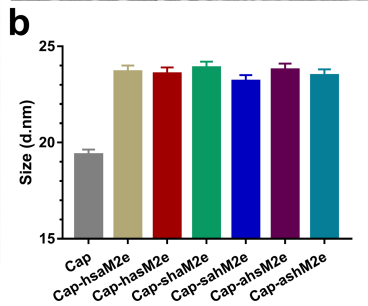

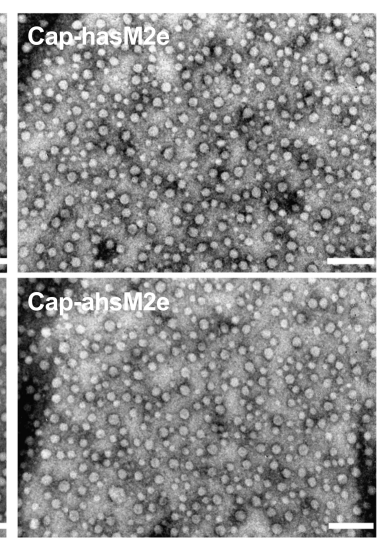

C

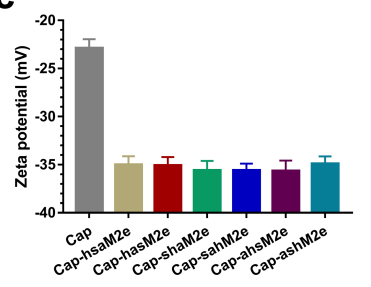



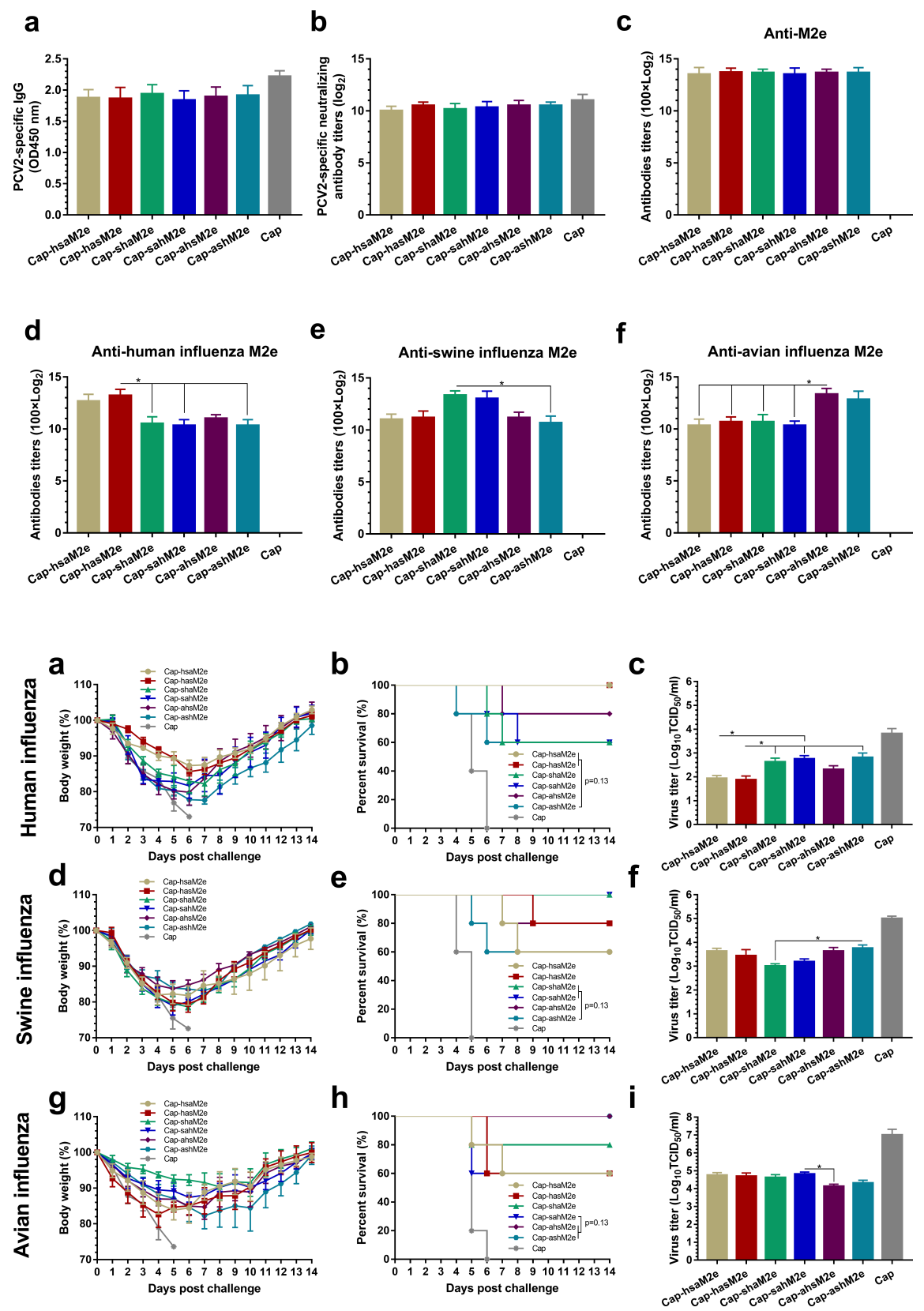